\title{
Clinical Significance of Electrocardiography Recordings From a Higher Intercostal Space for Detection of the Brugada Sign
}

\author{
Kiyoshi Nakazawa, MD; Tsuneharu Sakurai*; Akihiko Takagi, MD; Ryoji Kishi, MD; \\ Keizo Osada, MD; Osamu Miyazu, MD; Yoshiyuki Watanabe, MD; Fumihiko Miyake, MD
}

\begin{abstract}
Background The significance of higher intercostal space electrocardiography (HICS ECG) for the detection of the Brugada sign was investigated.

Methods and Results The subjects consisted of 113 cases (108 males, 5 females; mean age, $57 \pm 17$ years) with incomplete right bundle branch block type QRS morphology and ST-segment elevation $(>0.10 \mathrm{mV})$ in the right precordial leads. Obvious structural heart disease was not observed in any of the subjects. The $\mathrm{V}_{1-3}$ leads of the standard 12-lead ECG and the HICS ECG were recorded in the supine position, and the amplitude of the terminal portion of the QRS (J-point) and ST-segment ( $80 \mathrm{~ms}$ from the J-point) were measured. In the HICS ECG, there was an increase in the area in which the Brugada sign was detectable (47 leads to 66 leads), and in cases with the Brugada sign, the amplitude of the J-point increased.
\end{abstract}

Conclusions The HICS ECG may be helpful for the detection of the Brugada sign. (Circ J 2004; 68: 10181022)

Key Words: Brugada syndrome; Electrocardiography; Higher intercostal space

B rugada syndrome is a cardiac sudden-death syndrome in healthy adults that is electrocardiographically characterized by a coved-type ST-segment elevation in the right precordial leads of the electrocardiography (ECG). This strange ST-segment elevation is usually called the 'Brugada sign' and it indicates the diagnosis of Brugada syndrome (BS), which is considered to be a predictor of ventricular fibrillation! A saddleback-type ST-segment elevation is often detected in the right precordial leads in the clinical situation, but it is unknown whether it is related to BS. Recently, ECG recorded from a higher intercostal space (HICS ECG), after the administration of procainamide, has been reported as useful for the detection of the Brugada sign in survivors of sudden unexplained death syndrome and their relatives? In this study, the clinical significance of HICS ECG in cases with the Brugada sign and the saddleback-type was investigated.

\section{Methods}

\section{Subjects}

The study group consisted of 113 subjects (108 males, 5 females; mean age $57 \pm 17$ years) with an incomplete right bundle branch block type QRS morphology and ST-segment elevation $(>0.10 \mathrm{mV})$ in the right precordial leads. These ECG findings were recorded between April 1998 and March 2002. There were 13 symptomatic cases with the

(Received March 24, 2003; revised manuscript received August 16, 2004; accepted August 19, 2004)

Division of Cardiology, Department of Internal Medicine, St. Marianna University School of Medicine and *Division of Laboratory Medicine, St. Marianna University Hospital, Kawasaki, Japan

Mailing address: Kiyoshi Nakazawa, MD, Division of Cardiology, Department of Internal Medicine, St. Marianna University School of Medicine, 2-16-1 Sugao, Miyamae-ku, Kawasaki 216-8511, Japan coved-type ST-segment elevation (symptomatic BS group; survivors of primary ventricular fibrillation or sudden cardiac death), 24 cases with the coved-type ST-segment elevation, but without symptoms (asymptomatic BS group), and 76 asymptomatic cases with the saddleback-type STsegment elevation (asymptomatic saddleback group).

Obvious structural heart disease was not observed in any of the subjects, as determined by chest X-ray, echocardiography and exercise testing. Further, some subjects (13 from the symptomatic BS group and 18 from the asymptomatic BS group) underwent coronary angiography and ventriculography, which revealed nonstructural heart disease.

\section{ECG Recordings}

The $\mathrm{V}_{1-3}$ leads of the standard 12-lead ECG and the HICS ECG were recorded in the supine position (recording speed $25 \mathrm{~mm} / \mathrm{s}$ ), and the amplitude of the terminal portion of the QRS (J-point) and ST-segment $(80 \mathrm{~ms}$ from the Jpoint) were measured. When J-point elevation $(>0.2 \mathrm{mV})^{1}$ in association with the coved-type ST-segment elevation occurred in the right precordial leads, the Brugada sign was defined as positive.

\section{Statistical Analysis}

The significance of the measured data was determined using the Student's t-test, and $\mathrm{p}<0.05$ was defined as significance.

\footnotetext{
Results

Number of ECG Leads in Which the Brugada Sign was Detectable

A comparison of the number of ECG leads in which the Brugada sign was detectable is shown in Fig 1 . The Brugada sign was detected in the $\mathrm{V}_{1}$ lead only of the 12-
} 


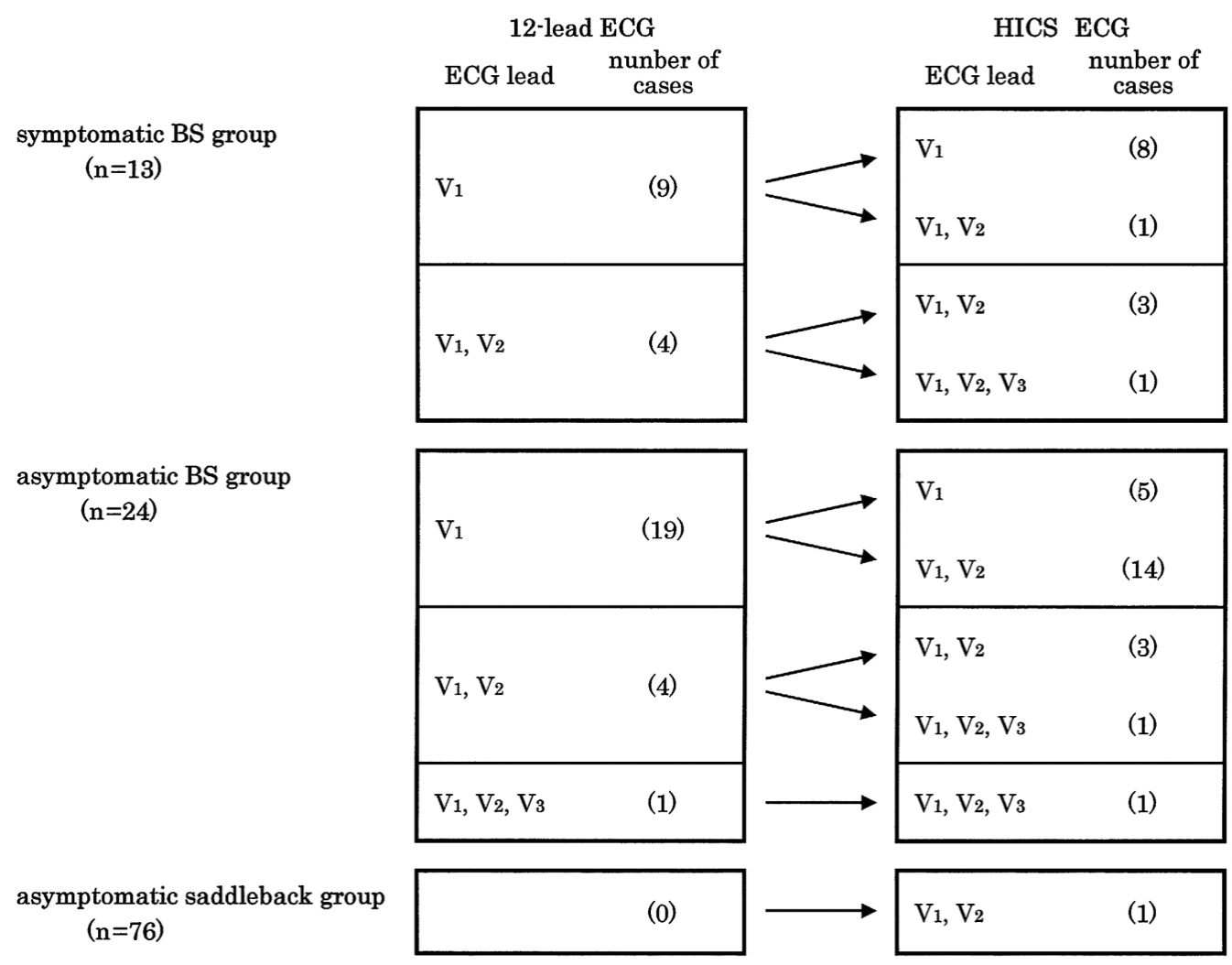

Fig 1. Detection of the Brugada sign in the $\mathrm{V}_{1-3}$ leads. Comparison of the recordings from the standard 12-lead ECG with those from leads located at a higher intercostal space (HICS ECG). BS, Brugada syndrome.

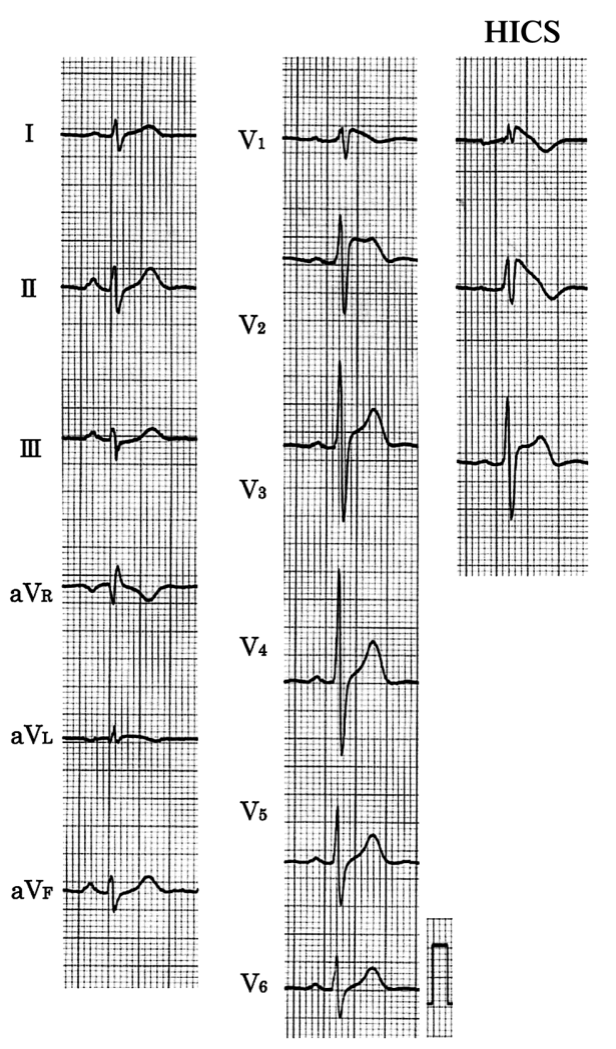

Fig 2. Case with the Brugada sign (67-year-old, male). The Brugada sign was detected in the $\mathrm{V}_{1}$ lead only of the standard 12-lead ECG, whereas in the HICS ECG, the area in which the sign was detectable spread to include both the $\mathrm{V}_{1}$ and $\mathrm{V}_{2}$ leads. HICS, higher intercostal space.

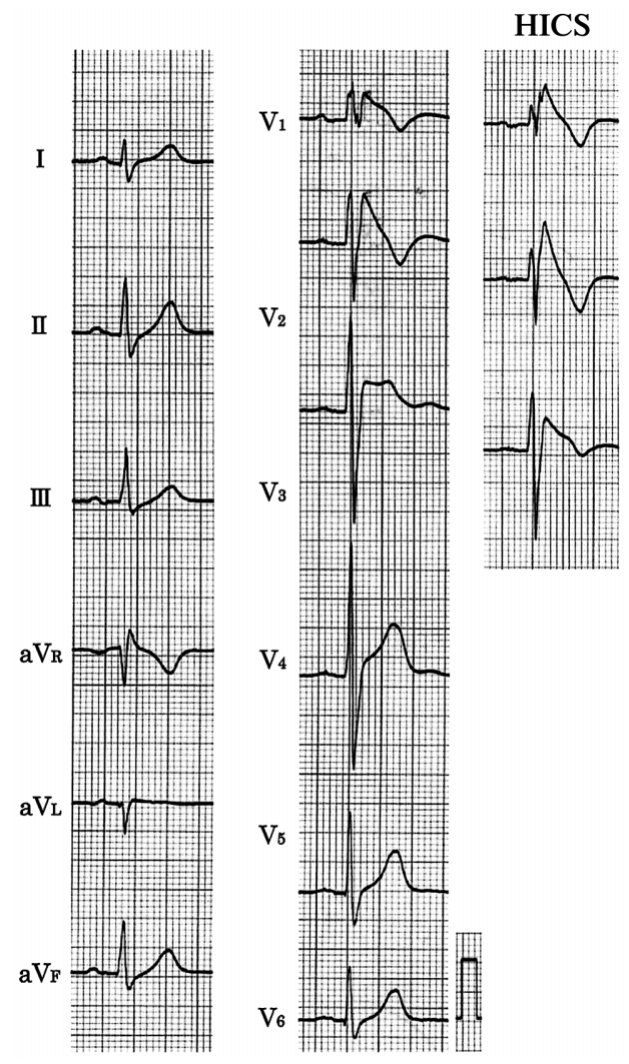

Fig 3. Case with the Brugada sign (52-year-old, male). The Brugada sign was detected in both the $\mathrm{V}_{1}$ and $\mathrm{V}_{2}$ leads of the standard 12-lead ECG, compared with the $\mathrm{V}_{1}, \mathrm{~V}_{2}$, and $\mathrm{V}_{3}$ leads on the HICS ECG. HICS, higher intercostal space. 


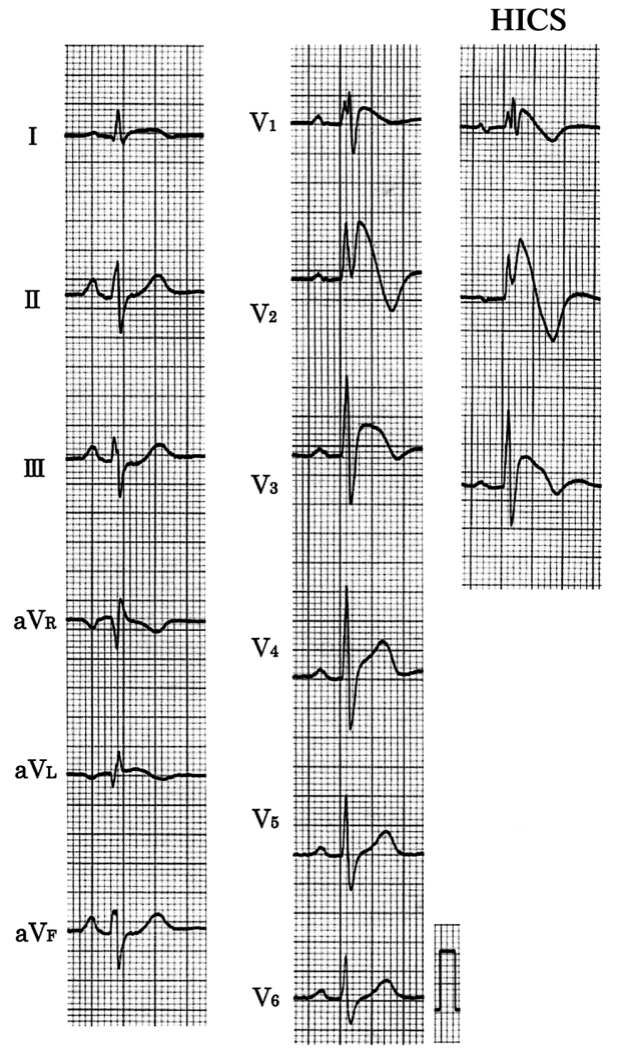

Fig 4. Case with the Brugada sign (51-year-old, male). The Brugada sign was detected in leads $V_{1-3}$ of the standard 12-lead ECG and of the HICS ECG. HICS, higher intercostal space.

lead ECG in 28 cases ( 9 symptomatic BS group, 19 asymptomatic BS group); however, in the HICS ECG, the Brugada sign was exhibited in the $\mathrm{V}_{1}$ lead only in 13 cases, and in the remaining 15 cases, the area in which the sign was detectable spread to include both the $\mathrm{V}_{1}$ and $\mathrm{V}_{2}$ leads (Fig 2). On the 12-lead ECG the Brugada sign was detected in both the $\mathrm{V}_{1}$ and $\mathrm{V}_{2}$ leads in 8 cases (4 symptomatic BS group, 4 asymptomatic BS group) and in 6 cases on the HICS ECG and in the remaining 2 cases, it was detected in the $\mathrm{V}_{1}, \mathrm{~V}_{2}$, and $\mathrm{V}_{3}$ leads (Fig 3). In 1 case the Brugada sign was detected in the $\mathrm{V}_{1-3}$ leads of both the 12-lead ECG and the HICS ECG (Fig 4).

Of the 76 cases with the asymptomatic saddleback-type, the Brugada sign was unmasked by the HICS ECG in only 1 case in which a $0.1 \mathrm{mV}$ elevation was detected in the $\mathrm{V}_{1}$ lead and $0.2 \mathrm{mV}$ elevation in the $\mathrm{V}_{2}$ lead (Fig 5).

Of the 113 cases, the Brugada sign was detected in 47 leads using the 12-lead ECG compared with 66 leads in the HICS ECG; however, in the asymptomatic saddleback group, the Brugada sign was rarely detected.

\section{Amplitude of the J-Point and ST-Segment}

A comparison of the amplitudes of the J-point and the ST-segment measured from the 12-lead ECG and the HICS ECG is shown in Fig 6.

At the J-point, no significant difference was observed in any of the subjects, but when they were divided into the different groups, the amplitude of the J-point in the asymptomatic saddleback-type group decreased in leads $\mathrm{V}_{1}$ and $\mathrm{V}_{2}\left(\mathrm{p}<0.01\right.$, respectively) and increased in leads $\mathrm{V}_{2}$ and $\mathrm{V}_{3}$

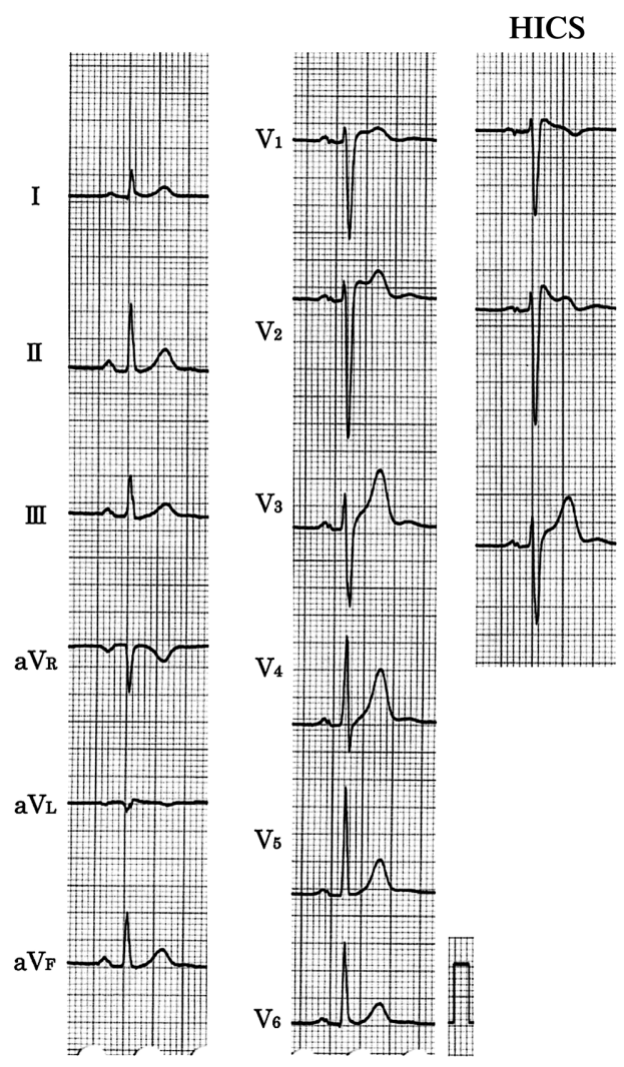

Fig 5. Case with the saddleback-type ST-segment elevation in the right precordial leads (42-year-old, male). The Brugada sign was unmasked in the $V_{1}$ and $V_{2}$ leads of the HICS ECG. HICS, higher intercostal space.

in the asymptomatic BS group ( $\mathrm{p}<0.05$, respectively). In the symptomatic BS group, the amplitude of the J-point increased in the $\mathrm{V}_{1}$ lead only $(\mathrm{p}<0.05)$.

In comparison with the 12-lead ECG, a significant decrease $(p<0.01)$ was observed in the amplitude of the ST-segment on the HICS ECG. When the subjects were divided into the symptomatic BS, asymptomatic BS, and asymptomatic saddleback groups, a significant decrease $(\mathrm{p}<0.01$, respectively) was also observed on the HICS ECG.

From these measurements, the following characteristics of the HICS ECG were identified: (1) a decrease in the amplitude of the J-point in the asymptomatic saddleback group; (2) an increase in the amplitude of the J-point in the symptomatic (only in $\mathrm{V}_{1}$ ) and asymptomatic (in $\mathrm{V}_{2}$ and $\mathrm{V}_{3}$ ) BS groups; and (3) a decrease in the amplitude of the STsegment in all groups (in $\left.\mathrm{V}_{1}-\mathrm{V}_{3}\right)$.

\section{Discussion}

BS was originally described by Brugada $\mathrm{P}$ and Brugada $\mathrm{J}$ in $1992^{3}$ and it appears that this syndrome originates from abnormal electrophysiological activity resulting from a genomic cardiac channelopathy. The prognosis of BS is poor, and at present an implantable cardioverter defibrillator is the only recommended treatment. Although there are many asymptomatic individuals, their management remains controversial. As a predictor for ventricular fibrillation (VF) susceptibility, Brugada et $\mathrm{al}^{1}$ supported the inducibility of VF in an electrophysiological study, but as that examination 

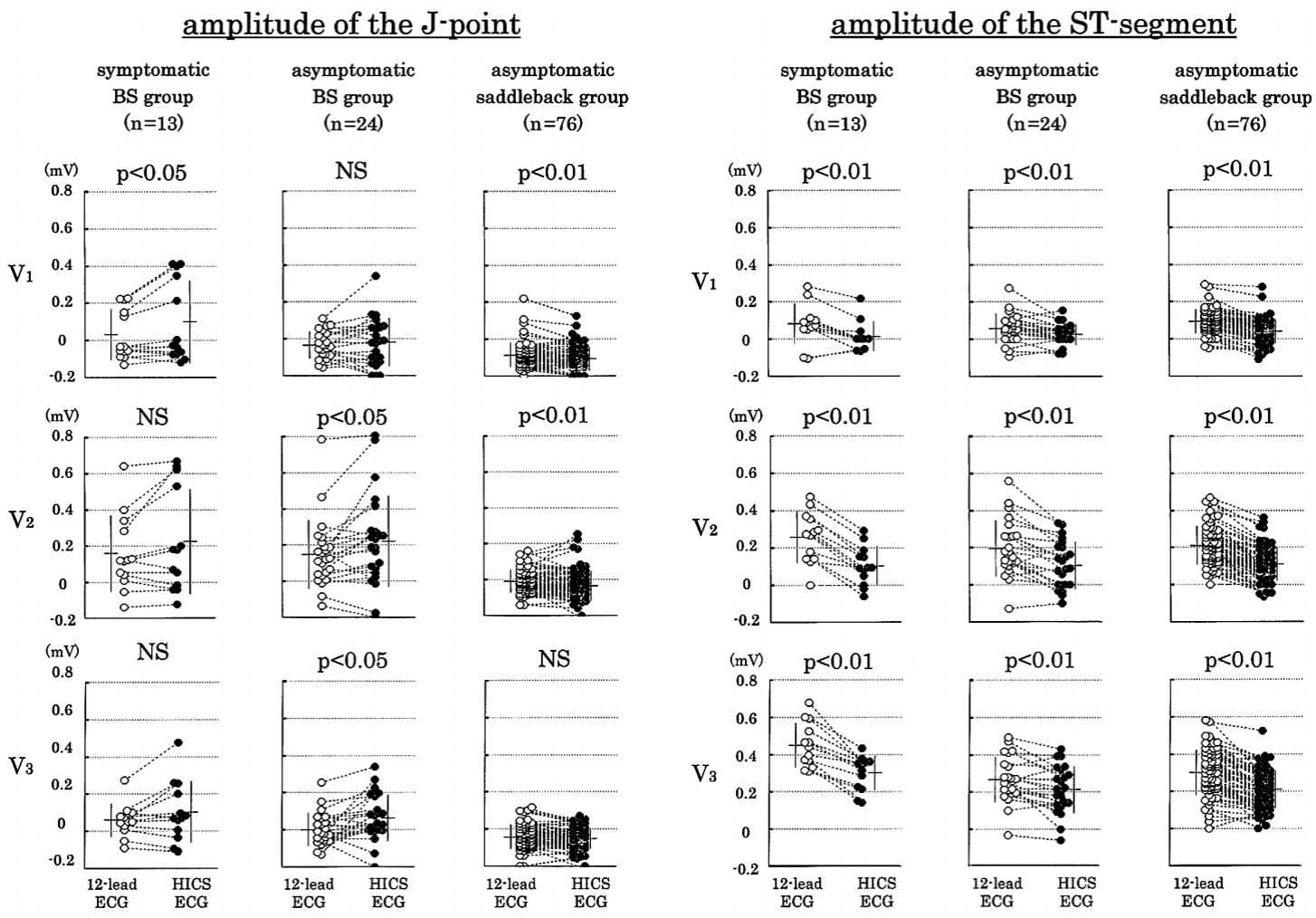

Fig 6. Comparison of the amplitude of the J-point and ST-segment at $80 \mathrm{~ms}$ from the J-point between recordings obtained from the $\mathrm{V}_{1-3}$ leads of the standard 12-lead ECG with those recorded on the HICS ECG. BS, Brugada syndrome; HICS, higher intercostal space.

does not easily identify all asymptomatic individuals, there is a need for a noninvasive method of predicting VF. Some noninvasive examinations have been proposed, but they have failed to show sufficient significance in distinguishing those with a high risk for BS. We have reported the significance of the dispersion of the activation recovery time, ${ }^{4}$ and that of ventricular late potentials ${ }^{5}$ and the association between RR interval and QT-interval ${ }^{6}$ have also been discussed. However, none of these examinations was sufficient for the prediction of VF susceptibility. At present, VF susceptibility is not consistently predicted by noninvasive examinations. If the patient agrees to an electrophysiological study, and VF inducibility is confirmed, an implantable cardioverter defibrillator is implanted.

Furthermore, problems with the diagnosis are not rare and because the Brugada sign can be $\operatorname{missed}^{1}$ (eg, when the ECG may show the saddleback-type ST-segment elevation and then return to normal), a method with higher probability is needed. Although the saddleback-type ST-segment elevation may sometimes be detected in a medical checkup, it is difficult to decide whether or not those cases could exhibit the Brugada sign in other situations. The Na channel blockers are widely used to unmask the Brugada sign, 7,8 but there is skepticism about whether the drug tolerance test can identify those individuals who have shown the asymptomatic saddleback-type during a medical check-up. Shimizu et al reported that body surface mapping studies using ECG recordings from the second and third intercostal spaces help detect the Brugada sign in patients with BS and in those with the Brugada sign unmasked by Na channel blockade? Most recently, Sangwatanaroj et al reported that ECG recordings from one intercostal space higher than usual was helpful in detecting the Brugada sign induced by procainamide administration in patients who had survived unexpected death syndrome in Thailand? We consider that it is easy to change the ECG recording site for an ECG during a medical check-up.

Our results showed that in patients with the Brugada sign observed on the 12-lead ECG, the sign can also be detected on the HICS ECG and furthermore, the area in which the Brugada sign is detectable is greater than when using the 12-lead ECG,; there was only 1 individual with the saddleback-type ST-segment elevation in whom the Brugada sign was unmasked by the HICS ECG.

The area in which the Brugada sign could be detected became greater when recording from a higher intercostal space than when using the 12-lead ECG in patients in whom the Brugada sign was observable in the 12-lead ECG. In the cases in which the Brugada sign was detected, the amplitude of the J-point increased and that of the STsegment decreased on the HICS ECG, most likely resulting in the coved-type ST-segment elevation. However, in the asymptomatic saddleback group, both the amplitude of the J-point and ST-segment tended to decrease on the HICS ECG. Therefore, if the Brugada sign is not detected in the 12-lead ECG in individuals with an increased amplitude of the J-point, recording a HICS ECG may be helpful to detect the Brugada sign.

In the cases in which the Brugada sign was detectable on the 12-lead ECG, the sign was always detectable on the HICS ECG and furthermore, the area in which the Brugada sign was detectable was greater than when using the 12 lead ECG. Because the HICS ECG is easy to use, it should always be performed and should be recorded first. 


\section{Conclusion}

The ability of the HICS ECG to detect the Brugada sign was investigated. The area in which the sign was detectable was greater when recording from a higher intercostal space than when using the 12-lead ECG. In cases with the Brugada sign, the amplitude of the J-point was increased and the amplitude of the ST-segment was decreased, therefore the Brugada sign was accentuated. We consider that HICS ECG is helpful for the detection of the Brugada sign.

\section{References}

1. Brugada J, Brugada R, Antzelevitch C, Towbin J, Nademanee K, Brugada P. Long-term follow-up of individuals with the electrocardiographic pattern of right bundle-branch block and ST-segment elevation in precordial leads V1 to V3. Circulation 2002; 105: $73-$ 78.

2. Sangwatanaroj S, Prechawat S, Sunsaneewitayakul B, Sitthisook S, Tosukhowong P, Tungsanga K. New electrocardiographic leads and the procainamide test for the detection of the Brugada sign in sudden unexplained death syndrome survivors and their relatives. Eur Heart J 2001; 22: 2290-2296.

3. Brugada P, Brugada J. Right bundle branch block, persistent ST segment elevation and sudden cardiac death: A distinct clinical and electrocardiographic syndrome: A multicenter report. J Am Coll Cardiol 1992; 20: $1391-1396$.
4. Nanke T, Nakazawa K, Arai M, Ryuu S, Osada K, Sakurai T, et al. Clinical significance of the dispersion of the activation-recovery interval and recovery time as markers for ventricular fibrillation susceptibility in patients with Brugada syndrome. Circ J 2002; 66: $549-552$.

5. Takagi A, Nakazawa K, Sakurai T, Nanke T, Miyake F. Prolongation of LAS40 (duration of the low amplitude electric potential component $(<40 \mu \mathrm{V})$ of the terminal portion of the QRS) induced by isoproterenol in 11 patients with Brugada syndrome. Circ J 2002; 66: $1101-1104$

6. Tohyo Y, Nakazawa K, Matsumoto N, Masui H, Sakurai T, Murayama M. Significance of QTc dispersion in right bundle branch block, ST segment elevation, and sudden cardiac death syndrome. In: Vardas PE, editor. EUROPACE '97. Bologna: Monduzzi Editor S. p. A.; 1997; 253-257.

7. Brugada P, Brugada R, Brugada J. Sudden death in patients and relatives with the syndrome of right bundle branch block, ST segment elevation in the precordial leads V(1) to V(3) and sudden death. Eur Heart J 2000; 21: 321-326.

8. Brugada R, Brugada J, Antzelevitch C, Kirsch GE, Potenza D, Towbin JA, et al. Sodium channel blockers identify risk for sudden death in patients with ST-segment elevation and right bundle branch block but structurally normal hearts. Circulation 2000; 101: 510515 .

9. Shimizu W, Matsuo K, Takagi M, Tanabe Y, Aiba T, Taguchi A, et al. Body surface distribution and response to drugs of ST segment elevation in Brugada syndrome: Clinical implication of eightyseven-lead body surface potential mapping and its application to twelve-lead electrocardiograms. J Cardiovasc Electrophysiol 2000; 11: $396-404$. 